\title{
单粒子四点翻转自恢复加固锁存器设计
}

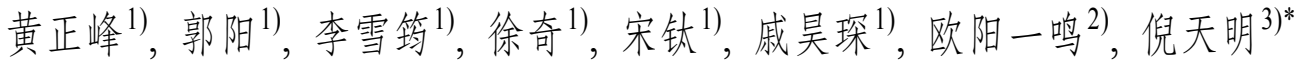 \\ 1) (合肥工业大学电子科学与应用物理学院 合肥 230001) \\ 2) (合肥工业大学计算机与信息学院 合肥 230001) \\ 3) (安徽工程大学电气工程学院 芜湖 241000) \\ (timmyni126@126.com)
}

\begin{abstract}
摘＼cjkstart要：为了容忍日益严重的单粒子多点翻转, 提出了一种能够容忍单粒子四点翻转的加固锁存器— QNURL (quadruple node upset recovery latch). 该锁存器包含 40 个同构的双输人反相器, 形成 $5 \times 8$ 的阵列结构, 构建了 多级过滤的容错机制. 通过有效地利用双输人反相器的单粒子过滤特性, 当任意 4 个内部状态节点同时发生翻转时, 都可以被多级过滤机制消除，自动恢复到正确值. PTM $32 \mathrm{~nm}$ 工艺下的仿真结果表明，与现有的 4 种单粒子多点翻转 加固锁存器综合比较, 该锁存器的单粒子四点翻转自恢复比率高达 $100 \%$, 延迟平均降低了 $86.02 \%$, 功耗延迟积 (power delay product, PDP) 平均降低了 78.94\%, 功耗平均增加了 $59.09 \%$, 面积平均增加了 $4.63 \%$. 文章最后对结构 进行了衍生, 提出了容忍 $N$ 点翻转的 $(N+1) \times 2 N$ 结构框架.
\end{abstract}

关键词: 单粒子翻转; 抗辐射加固设计; 四点翻转; 双输人反相器; 软错误自恢复

中图法分类号: TP391.41 DOI: 10.3724/SP.J.1089.2021.18573

\section{Single-Event Quadruple-Upset Self-Recoverable Latch Design}

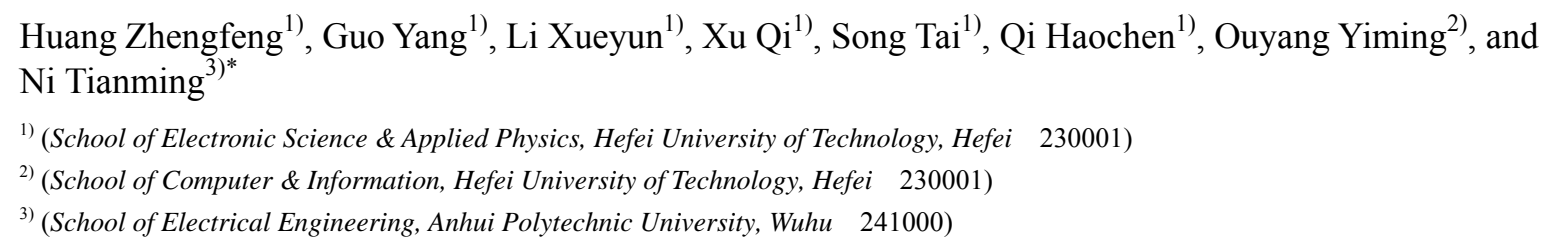

\begin{abstract}
For the sake of the requirement to tolerate increasingly serious single event multimode upset, a kind of radiation hardened latch which can tolerate single event quadruple node upset: QNURL (quadruple node upset recovery latch) is proposed. The QNURL consists of 40 isomorphic dual-input inverters, forming a $5 \times 8$ array structure, and constructing a fault-tolerant mechanism of multi-level filtering. The single-particle filtering characteristics of the dual input inverter are effectively utilized. When any 4 internal state nodes upset at the same time, they can be eliminated by the multi-level filtering mechanism and automatically restored to the correct value. The experimental results under the PTM $32 \mathrm{~nm}$ process show that compared with the existing four kinds of single event multimode up set radiation hardened latches, the single event quadruple node up set self recovery rate of the QNURL is as high as $100 \%$, and the average delay is reduced by $86.02 \%$. The average power delay product (PDP) is reduced by $78.94 \%$. The average power consumption is
\end{abstract}

收稿日期: 2020-08-13; 修回日期：2020-09-21. 基金项目：国家自然科学基金(61874156，61874157，61904001，61904047); 安徽省自然科学基金(1908085QF272). 黄正峰(1978一), 男, 博士，教授，硕士生导师，CCF 高级会员，主要研究方向为硬件容错; 郭阳(1995一), 男, 硕士研究生, 主要研究方向为硬件容错; 李雪筠(1995-), 女, 硕士研究生, 主要研究方向为硬件容错; 徐奇 (1991一), 男, 博士, 讲师, 主要研究方向为 3D 芯片测试与容错; 宋钛 (1981一), 男, 博士研究生, 主要研究方向为内建自测 试; 戚吴琛(1981-), 女, 硕士, 副教授, 主要研究方向为硬件安全; 欧阳一鸣(1963-), 男, 博士, 教授, 博士生导师, CCF 高级 会员, 主要研究方向为 NoC 测试与容错; 倪天明(1991一), 男, 博士, 讲师, 论文通讯作者, 主要研究方向为 3D 芯片测试与容错. 
increased by $59.09 \%$, and the average area is increased by $4.63 \%$. In this paper, the structure $(N+1) \times 2 N$ is derived, and also proposed a structure framework which can tolerant $N$-nodes upset.

Key words: single event upset; radiation hardening by design; quadruple node upset; dual input inverter; soft error self-recovery

电平敏感型锁存器常用来构成主从边缘触发 器，它会影响整个系统的安全性. 传统的静态锁存 器在太空会发生单粒子翻转(single event upset, SEU), 使存储的值发生跳变. SEU 不会造成器件发 生永久性的损害, 所以被称为软错误. 抗辐射加固 (radiation hard by design, RHBD)是一种通过冗余 技术保护 SEU 的方法 ${ }^{[1-6]}$, 著名的 RHBD 技术如三 模气余 ${ }^{[7]}$ 和 $\mathrm{DICE}^{[8]}$, 在大特征尺寸下能很好消除 仅在单个节点上的辐射引起的故障. 然而, 在深亚 微米和纳米技术中, 单粒子多节点翻转 (multiple nodes upset, MNUs) 对电子电路构成严重威胁. 它 们与 SEU 的主要不同是多节点同时变化, 而仅针 对 SEU 的传统 RHBD 方法可能无法完全消除这种 威胁.

另外单粒子瞬态(single event translate, SET)也 是辐射环境下工作的锁存器软错误来源之一. 它 会以脉冲的形式在组合逻辑中传播，满足主从触 发器的建立保持时间后, 就会被捕获. 在高频率下 工作的电路, SET 是软错误的主导因素.

本文主要提出了一种适用于 MNUs 的加固锁 存器设计框架; 并以现行特征尺寸下要求较高的
四点翻转加固结构 QNURL 为例子, 介绍了该框架 的加固原理和性能对比. 该框架主要以双输人反 相器构成, 还可以根据实际性能要求, 用不对称的 $C$ 单元替换. 最后在输人端添加一个延迟单元时, 这个锁存器可以进一步加固以容忍 SET.

本文的其余部分组织如下. 第 1 节介绍最新的 抗辐射加固结构. 第 2 节介绍 QNURL 结构的物理 组成、工作原理、加固原理和仿真分析. 第 3 节介 绍性能对比和一些其他的衍生 RHBD 方案. 第 4 节给出了结论.

\section{1 已有的加固设计}

\subsection{TNURL 结构}

TNURL 结构如图 1 所示 ${ }^{[9]}$, 它利用 $C$ 单元的 高阻态保证内部其他节点的稳定，最终通过节点 间的反馈，自恢复发生翻转的节点. 但是该结构实 现复杂, 电路的面积和功耗开销非常大. 另外, 该 结构只能容忍三点翻转, 并不能容忍四点翻转. 在 集成电路特征尺寸不断变小的情况下, 可能无法 在高辐射环境下工作.

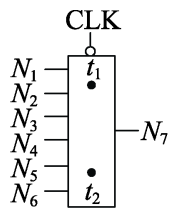

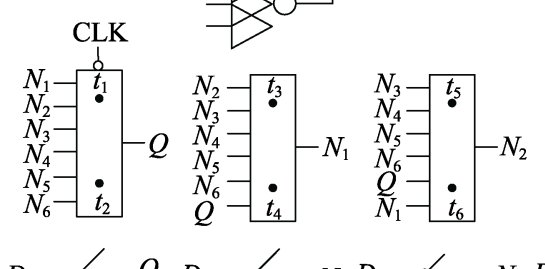
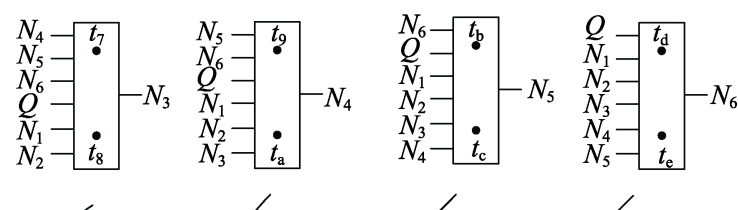

图 1 TNURL 电路

\subsection{TNU-Latch 结构}

TNU-Latch 结构如图 2 所示, 它同样利用 $C$ 单元的高阻态，保持内部节点的稳定. 但是，该 电路内部节点会长时间处于高阻态，将使其受到 漏电流的影响. 另外, 该结构的面积以及延迟开 销很大, 而且只能容忍三点翻转, 不能容忍四点
翻转, 在对性能有一定要求的高辐射环境下可能 无法正常工作.

\subsection{TNUTL 结构}

如图 3 所示, TNUTL 结构 ${ }^{[10]}$ 是由 6 个 $C$ 单元 组成, 其利用 $C$ 单元的保持功能维持 $Q$ 节点的稳 定. 但是该结构内部并没有形成锁存环结构, 不能 


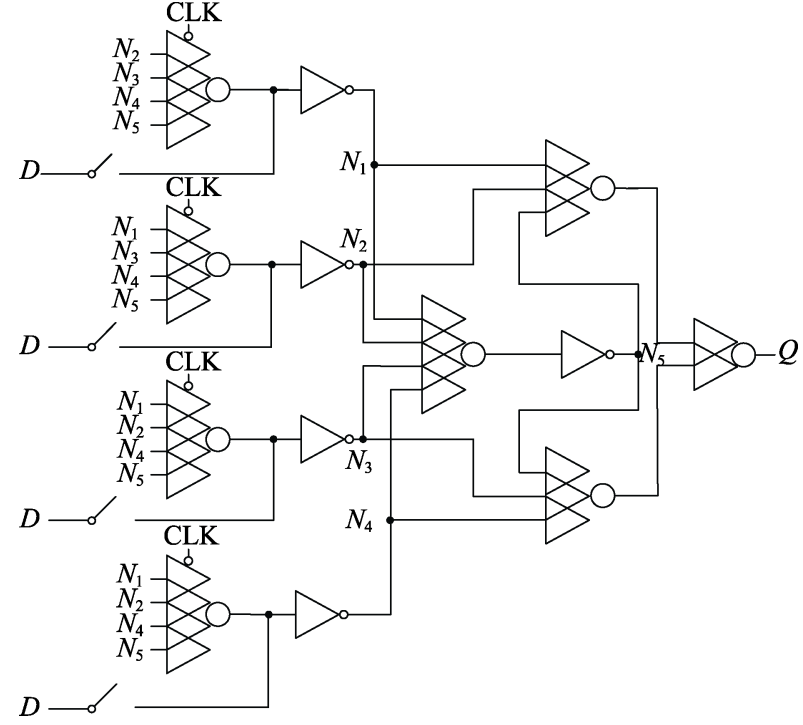

图 2 TNU-Latch 电路

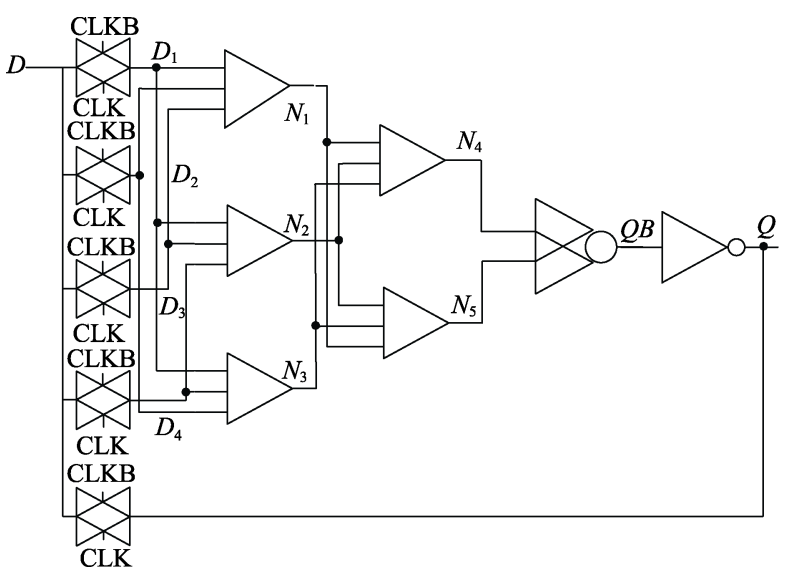

图 3 TNUTL 电路

静态存储数据, 只能依靠节点电容动态锁存数据, 存储的数据会受到漏电流的影响. 同时该结构只能 容忍三点翻转, 在高辐射环境下可能无法正常工作.

\subsection{LCTNUT 结构}

如图 4 所示, LCTNUT 结构 ${ }^{[11]}$ 利用 $C$ 单元以及 双输人反相器的保持功能, 不仅使输出 $Q$ 节点稳 定, 还能保持其他内部节点的稳定状态. 该结构中 加人了一些双输人反相器代替 $C$ 单元, 减小了结 构的面积以及延迟开销. 但是该结构并没有考虑 双输人反相器的 PMOS 以及 NMOS 都导通的情况, 同时它也只能容忍三点翻转, 且不能在高辐射环 境下工作

\section{2 本文工作概述}

\section{1 本文设计结构}

为了解决现阶段没有容忍四点翻转的锁存器
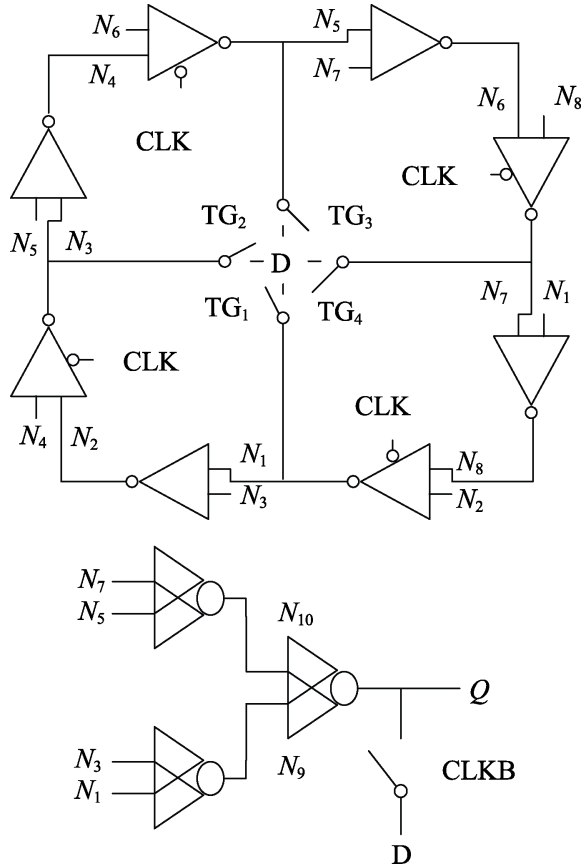

图 4 LCTNUT 电路

设计, 以及现存的设计的 PDP 和延迟较大的情况. 本文提出了一种能够自恢复四点翻转的锁存器设 计. 该设计以双输人反相器作为基本器件, 为了避 免双输人反相器的 PMOS 以及 NMOS 都导通时会 输出亚稳态的情况, 双输人反相器中采用的 NMOS 的尺寸较大, 驱动能力也相应较大, 输出值 会趋于稳定 ${ }^{[12]}$. 同时这样的设计使输人到 NMOS 的逻辑值改变时, 才改变整个双输人反相器的输 出值. 该设计如图 5 所示, 其中包含 10 个传输门 $\left(\mathrm{TG}_{1} \sim \mathrm{TG}_{10}\right)$ 和 40 个双输人反相器 $\mathrm{DI} i j$. 双输人反 相器排列成 5 行 8 列, 每个双输人反相器以器件所 在的列编号为 $i(i=0,1,2,3,4,5,6,7)$, 行编号为 $j(j=0,1,2,3,4)$. 列数 $i$ 为模 8 加运算, 运算值对 8 取模为最终值; 例如, 列数 $i$ 为 $5, i+7$ 表示 $(i+7) \bmod 8$, 结果为 4 . 行数 $j$ 为模 5 加运算, 运 算值对 5 取模为最终值; 例如, 列数 $i$ 为 $2, i+3$ 表 示 $(i+3) \bmod 5$. 内部的双输人反相器之间采用 2 种连接方式, 其中图中第一列人反相器的 PMOS 被 $\mathrm{DI}_{(i+7)(2+j)}$ 驱动, NMOS 被 $\mathrm{DI}_{(i+7)(1+j)}$ 驱动. 其余 几列双输人反相器的 NMOS 和 PMOS 都被 $\mathrm{DI}_{(i+7) j}$ 驱动和 $\mathrm{DI}_{(i+7)(j+1)}$ 驱动. 通过这样的连接方式, 使 每行上的二输人反相器的 NMOS 输人都来自同一 行, 当电路中发生 SEU 时, 错误逻辑值只会在同 一行上传播，同时消除共模故障.

该锁存器的工作原理如下. 当 CLK 为 1 时, 传 输门 $\mathrm{TG}_{1}, \mathrm{TG}_{2}, \mathrm{TG}_{3}, \mathrm{TG}_{4}, \mathrm{TG}_{5}$ 打开, 传输门 $\mathrm{TG}_{6}$, 


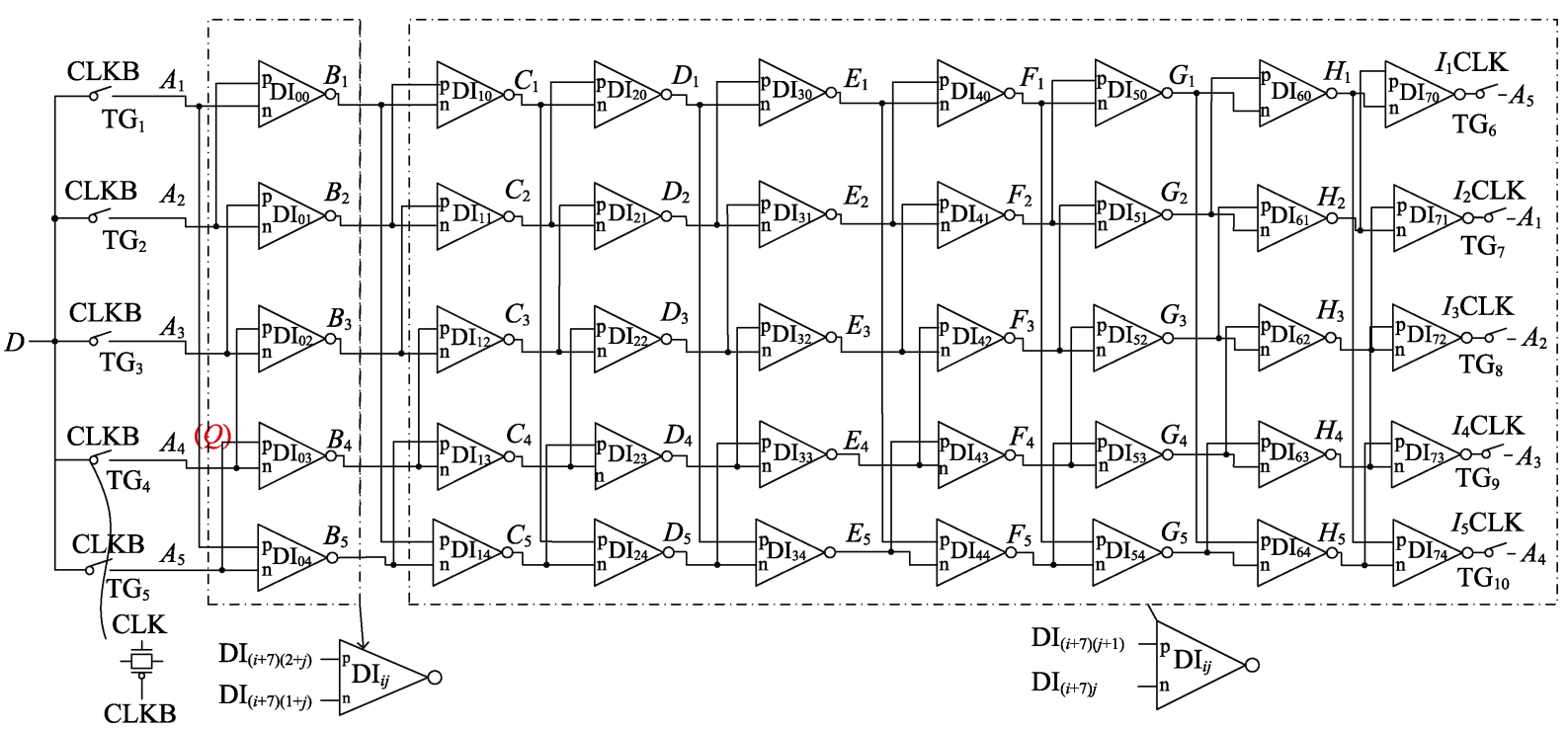

图 5 本文结构

$\mathrm{TG}_{7}, \mathrm{TG}_{8}, \mathrm{TG}_{9}, \mathrm{TG}_{10}$ 关闭, 锁存器工作在透明 期. 输人 $D$ 上的数据通过传输门进人锁存器内部 $A_{1}, A_{2}, A_{3}, A_{4}, A_{5}$ 节点, 并通过 $A_{5}$ 节点将 $D$ 输出. 而且锁存器反馈环中的传输门关闭, 减少了由于电 路竞争产生的功耗. 当 $\mathrm{CLK}$ 为 0 时, 传输门 $\mathrm{TG}_{1}$, $\mathrm{TG}_{2}, \mathrm{TG}_{3}, \mathrm{TG}_{4}, \mathrm{TG}_{5}$ 关闭, 传输门 $\mathrm{TG}_{6}, \mathrm{TG}_{7}$, $\mathrm{TG}_{8}, \mathrm{TG}_{9}, \mathrm{TG}_{10}$ 打开, 锁存器进入锁存期. 此时外 部数据 $D$ 不能进入锁存器内部, 锁存器内部的反 馈环开始工作, 通过双输人反相器互相连接形成 反馈, 静态保持数据.

\section{2 容错原理}

当电路结构可以自恢复 4 个节点同时翻转造 成的故障时，它一定可以自恢复 3 个节点以及更少 节点同时翻转造成的故障. 所以本文仅讨论电路 中 4 个节点同时发生翻转的情况.

按照电路中翻转的节点传播过程中的关系, 将 加固原理分为 4 种分别分析, 并且采用 Hspice 工具 进行故障注人分析. 仿真条件如下：采用 $32 \mathrm{~nm}$ PTM 工艺, 温度设置为室温 $25{ }^{\circ} \mathrm{C}$, 供电电压设置 为 $0.9 \mathrm{~V}$. 为了便于故障注人, 将时钟周期设置为

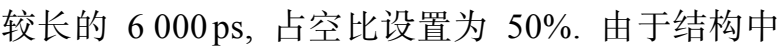
节点所处的位置不同，相同剂量的能量造成的翻 转情况不同, 因此为了实验的准确, 每个翻转的节 点注人的能量, 最小设为该节点逻辑值翻转全摆 幅的能量值.

（1）假设电路中的任意 4 个节点由于电荷共享 同时发生了翻转, 并且这 4 个错误节点的传播路径 没有发生交叠，即不同错误值在电路中传播时没 有经过相同的双输人反相器. 则这 4 个翻转节点之
间就没有任何影响，相当于发生了 4 次单点翻转. 基于电路的对称性，只需考虑一种情况，选择取 $A_{1}, A_{3}, E_{1}, E_{3}$ 节点进行 0 到 1 的故障注人进行分析. 如图 6 所示, $A_{1}$ 节点发生 0 到 1 翻转后, 造成 $B_{1}$ 节 点发生继发的 1 到 0 翻转; 之后双输人反相器的高 阻态将阻断错误逻辑的传播. 同样, $A_{1}$ 节点发生 1 到 0 翻转后, 错误逻辑值也会被高阻态阻断传播, 并且正确逻辑值的反馈使 $A_{1}$ 节点的逻辑值迅速变 为正确值. 在其余 3 个节点上错误逻辑的传播与之 类似，都迅速自恢复为正确值且之间没有影响.

（2）考虑任意 2 个翻转节点互相影响的情况. 4 个翻转的节点包含 2 个互相影响的节点和 2 个相对 独立的节点. 按照上文的分析, 2 个相对独立的节 点会迅速自恢复为正确值，而 2 个互相影响的节点 会因为错误逻辑值的组合使错误值传播到更多节 点. 按照节点影响关系将 2 个互相影响的节点分为 3 组: 第 1 组为发生翻转节点对的位置位于同一行, 第 2 组为发生翻转的节点对位于同一列, 第 3 组为 发生翻转的节点对位于不同行与不同列上. 对这 3 种情况分别进行遍历分析实验，可知发生在同一 列的节点会传播最多的节点. 此时取 $A_{1}, A_{2}, C_{3}, E_{4}$ 节点进行分析. 如图 6 所示, 在输人 $D$ 为 0 的情况 下， $A_{1}$ 和 $A_{2}$ 节点发生 0 到 1 翻转，错误值会一直传 播最终使 $B_{1}, B_{2}, C_{1}, D_{1}$ 节点翻转; 并且由于 $E_{1}$ 处 的高阻态使 $E_{1}$ 处于保持状态, 维持正确状态, 使 错误信号不再传播. 而 $C_{3}$ 和 $E_{4}$ 节点传播相对独 立, 都在传播一个器件后自恢复到正确值. 在输人 $D$ 为 1 的情况下, $A_{1}$ 和 $A_{2}$ 节点发生 1 到 0 翻转，错 误值会一直传播最终使 $B_{1}$ 和 $C_{1}$ 节点翻转; 并且同 
样由于 $D_{1}$ 处的高阻态使 $D_{1}$ 处于保持状态，维持正 确状态，使错误信号不再传播. 这种情况下的其他 翻转节点组合在发生翻转时，同样会有类似的过 程恢复正确值.

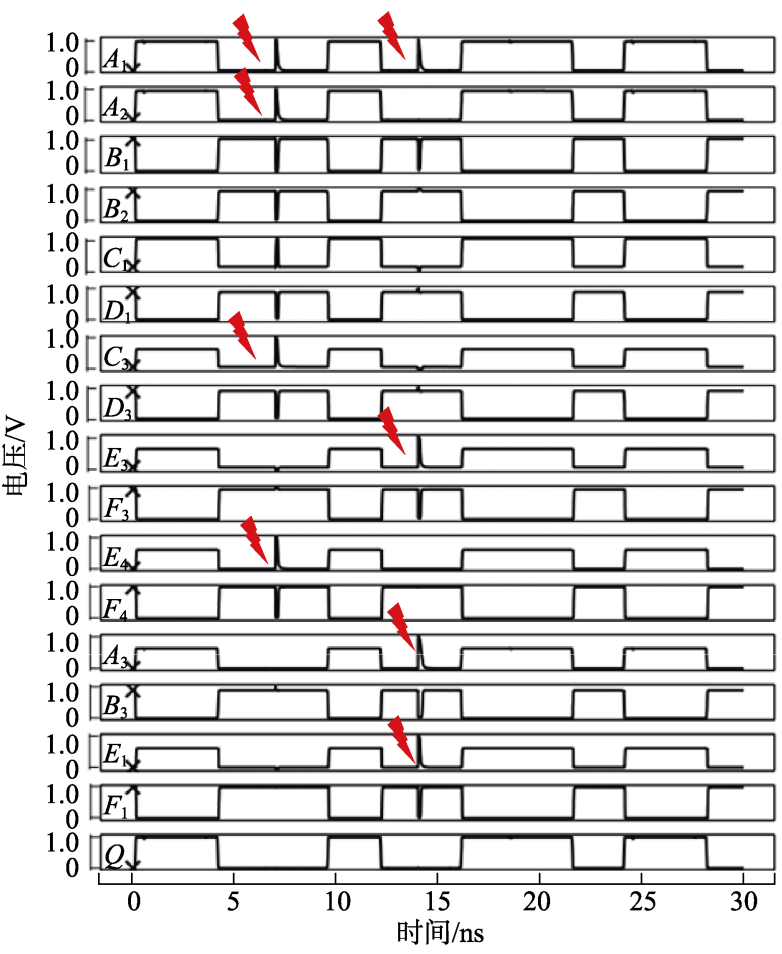

图 6 翻转节点中 3 个节点以下互相影响仿真故障注人

（3）考虑任意 3 个翻转节点互相影响的情况. 这种情况下, 4 个翻转的节点包含 3 个互相影响的 节点和 1 个相对独立的节点. 同样根据上文的分 析，相对独立发生翻转的节点会迅速恢复到正确 值, 只需考虑互相影响的 3 个翻转节点的情况. 这 种情况下，节点组合很多. 根据上文的分析进行遍 历实验，同样发生翻转的节点对位于同一列会产 生最多的翻转节点. 如图 7 所示, 取传播过程会相 互影响的 $A_{1}, A_{2}, A_{3}$ 节点以及 1 个相对独立的节点 $E_{3}$ 进行分析，在输人 $D$ 为 0 的情况下, 4 个节点发 生 0 到 1 翻转, 错误值会一直传播最终使 $B_{1}, B_{2}$ $B_{3}, C_{1}, C_{2}, D_{1}, C_{2}, E_{1}, F_{1}$ 以及 $F_{3}$ 节点翻转; 并且 $F_{1}$ 和 $F_{3}$ 处的错误值会使 $G_{1}$ 和 $G_{3}$ 处于高阻态, 维 持正确状态，使错误信号不再传播. 错误信号短暂 停留后会自恢复为正确值，同时高阻态消失. 在输 人 $D$ 为 1 的情况下, 4 个节点发生 1 到 0 翻转，错 误值会一直传播最终使 $B_{1}, B_{2}, C_{1}, C_{2}, D_{1}, E_{1}$ 以及 $F_{3}$ 节点翻转; 并且由于 $F_{1}$ 和 $F_{3}$ 处的高阻态使 $F_{1}$ 和 $F_{3}$ 处于正确状态，错误信号不再传播. 这种情况 下的其他翻转节点组合在发生翻转时，同样会有

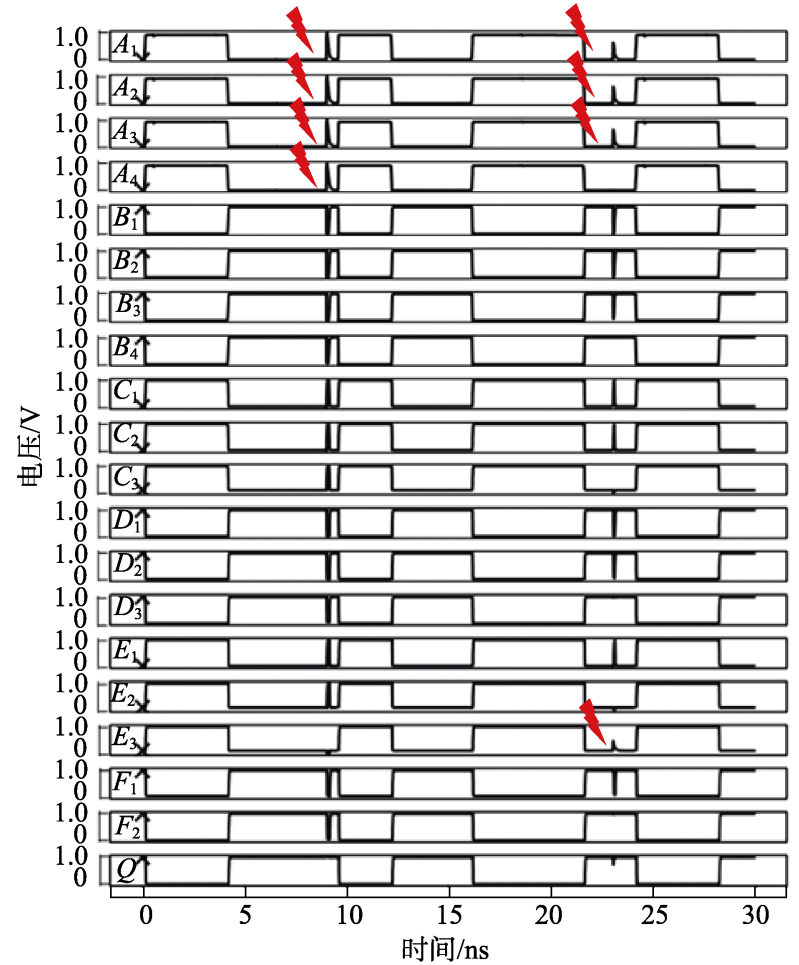

图 7 翻转节点中 3 个节点以上互相影响仿真故障注人

类似的过程恢复正确值.

（4）最后考虑 4 个节点的传播路径互相影响的 情况. 同样根据上文的分析同时进行遍历实验，发 生翻转的节点对位于同一列会产生最多的翻转节 点. 如图 7 所示, 取 $A_{1}, A_{2}, A_{3}, A_{4}$ 节点进行分析, 在输人 $D$ 为 0 的情况下, 4 个节点发生 0 到 1 翻转, 错误值会一直传播最终使 $B_{1}, B_{2}, B_{3}, B_{4}, C_{1}, C_{2}$, $C_{3}, D_{1}, D_{2}, D_{3}, E_{1}, E_{2}, F_{1}, F_{2}, G_{1}, H_{1}$ 节点翻转; 并 且由于 $I_{1}$ 处的高阻态, 使 $I_{1}$ 处于保持状态, $I_{1}$ 节 点交叉连接到了维持正确值的 $A_{5}$ 节点, 将处于正 确状态, 使错误信号不再传播. 在输人 $D$ 为 1 的情 况下, 情况与上述过程类似. 也会自恢复为正确值.

综上所述, 以上 4 种分析包含了电路中 4 个翻 转节点的所有组合. 由分析和仿真结果可知, 提出 的结构可以迅速自恢复电路中发生翻转的所有节 点. 而且由于整个结构是自恢复错误节点, 因此即 使错误节点发生的位置靠近输出节点, 造成输出节 点的翻转, 节点也会迅速自恢复为正确值, 只会产 生一个短暂的毛刺, 不会造成逻辑值长时间翻转.

另外, 考虑注人电荷量不同对电路造成的影 响不同, 在实验中不断加大故障注人能量等级, 考 查电路的加固能力. 实验结果如图 8 所示, 可以看 到在 3 次大剂量的故障注人实验中, 翻转节点的电 压值从正确值 $0 \mathrm{~V}$ 分别翻转到 $1.5 \mathrm{~V}, 1.6 \mathrm{~V}, 2.1 \mathrm{~V}$. 每 


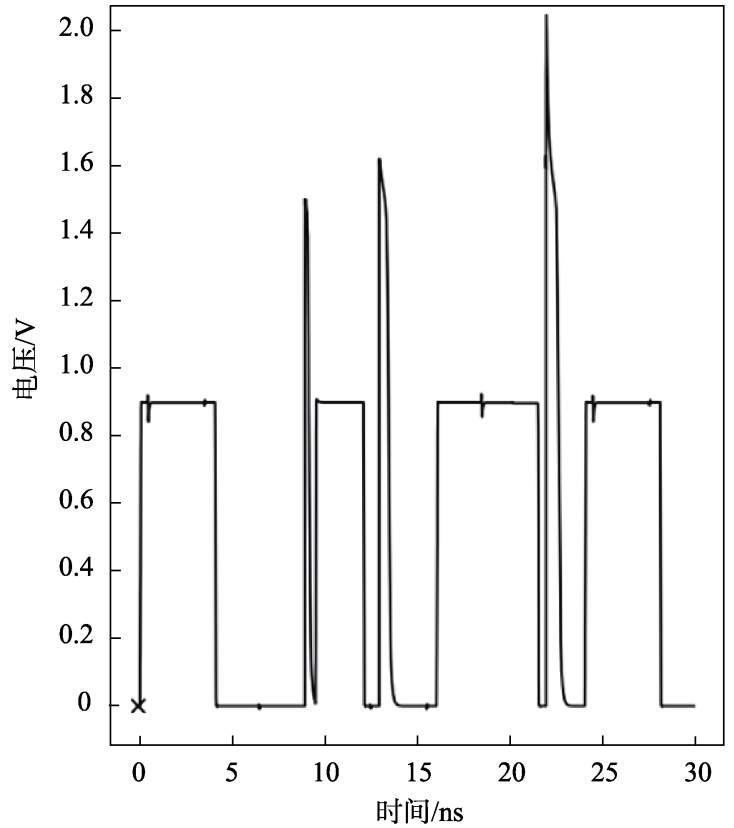

图 8 不同能量等级的故障注人实验

次翻转后的错误逻辑值都迅速恢复到正确值，只 是随着能量的提升，恢复时间逐渐增加.

\section{3 对比与衍生}

将本文设计与已有的加固结构进行多方面的 比较. 仿真条件仍然保持本文第 2.2 节的设定, 其 中延迟是指在透明期时输人 $D$ 到 $Q$ 的延迟; 功耗 是指在同样的输人下 $40 \mathrm{~ns}$ 时间内整个电路产生的 平均功耗; 结构面积采用宽长比最小的晶体管 (unit size transistors, UST) 数目之和权衡 ${ }^{[13]}$, 即

$$
\mathrm{UST}=\sum_{i=1}^{n}(W / L)_{i}
$$

\section{1 性能与开销比较}

QNURL 结构为了提升加固能力, 不可避免地 增大了部分开销, 但是结构中采用多种方法减小 开销. 首先, QNURL 为了减小整个电路的功耗和 面积，没有采用 $C$ 单元，使用了开销更小的双输人 反相器, 并且在电路中加人了一些钟控模块, 显著 减小了整个电路的功耗开销. 另外，为了减小延 迟，采用第 3 路旁路将逻辑值输出. 从表 1 中可以 看到, 与现有的结构比较, QNURL 结构的功耗虽 是最大的，但是仅比其余结构整体功耗的平均值 大了 $59.09 \%$; 在延迟方面，它仅次于 LCTNUT 结 构. PDP 能够很好地衡量一个电路的功耗延迟特 性, 表 1 同样比较了这些结构 PDP, 由于 QNURL 结构采用了多个措施减小功耗以及延迟，使其
表 1 不同结构性能与开销对比

\begin{tabular}{lcccc}
\hline \multicolumn{1}{c}{ 锁存器 } & 功耗/ $\mu \mathrm{W}$ & 延迟/ps & PDP/aJ & UST \\
\hline TNURL $^{[9]}$ & 1.7 & 14.7 & 25.6 & 390 \\
TNUTL $^{[10]}$ & 0.7 & 38.1 & 27.8 & 114 \\
TNU-Latch $^{[6]}$ & 1.3 & 118.6 & 154.5 & 222 \\
LCTNUT $^{[1]}$ & 0.7 & 6.1 & 4.1 & 138 \\
QNURL $^{11]}$ & 1.8 & 6.2 & 11.2 & 226 \\
\hline
\end{tabular}

PDP 也处于相对较小值.

为了直观地观察这些结构开销的相对变化, 表 2 列出了 QNURL 结构与对比结构的相对开销对 比, 其中, $\Delta=100 \% \times$ (QNURL 结构/对比锁存器). 若值为正, 则意味着 QNURL 的该项指标优于对比 结构; 若值为负, 则意味着 QNURL 的该项指标劣 于对比结构. 从表 2 可以看到, QNURL 结构在电路 延迟方面仅次于 LCTNUT, 但仅增加了 $1.64 \%$, 延 迟比平均值提升了 $86.02 \%$; 同时 PDP 也平均提升 了 $78.94 \%$, 只增加了 $4.63 \%$ 的面积开销和 $59.09 \%$ 的功耗.

表 2 不同结构相对开销比较

\begin{tabular}{lrrrr}
\hline \multicolumn{1}{c}{ 结构 } & \multicolumn{1}{c}{$\Delta \mathrm{TP} / \%$} & $\Delta t_{\mathrm{cq}} / \%$ & $\Delta \mathrm{PDP} / \%$ & $\Delta \mathrm{UST} / \%$ \\
\hline TNURL $^{[9]}$ & -5.88 & 57.82 & 56.40 & 42.05 \\
TNUTL $^{[10]}$ & -157.14 & 83.73 & 59.86 & -98.25 \\
TNU_Latch $^{[6]}$ & -38.46 & 94.77 & 92.78 & -1.80 \\
LCTNUT $^{[11]}$ & -157.16 & -1.64 & -172.20 & -63.77 \\
\hline 平均 & -59.09 & 86.02 & 78.94 & -4.63 \\
\hline
\end{tabular}

\section{2 加固能力比较}

一般在加固结构中都会存在 $C$ 单元、双输人 反相器等基本单元, 这些内部单元在正常工作时 不会出现高阻态, 若输人节点的逻辑状态有错误, 就有可能产生高阻态. 在发生单粒子翻转时, 仅保 证输出正确的结构, 在内部可能会产生高阻态, 进 而存储单元中存储的逻辑值会受到漏电流的影响.

QNURL 最主要的功能是可以自恢复四点翻 转, 保证全部节点都正确. 表 3 比较了几种锁存器 的加固能力, 其中, ST(SEU-tolerant) 表示该结构 可以容忍 SEU, 即该结构在发生一个节点翻转时, 仅能够保证输出正确; SR(SEU-resilient)表示该结构 可以自恢复任意 SEU, 即该结构在发生任意一个节 点翻转时, 不仅能够保证输出正确, 还能保证内部 节点正确. 另外, DT(DNUs-tolerant), DR(DNUsresilient), TT(TNUs-tolerant), TR(TNUs-resilient), QT(QNUs- tolerant)和 QR(QNUs-resilient)依次表示 结构在发生任意 2 个、3 个、 4 个节点同时翻转时, 
仅保证输出正确，还是可以保证全部节点正确. 从 表 3 可以看出, 目前存在的结构都不能容忍 QNUs, 更不能自恢复 QNUs. QNURL 结构是第 1 种可以容 忍并自恢复 QNUs 的结构，所以该结构可以使用在 一些特别恶劣的环境中以及更小的特征尺寸下. 另外, QNURL 结构的一个非常重要的特征是不会 受到漏电流的影响, 这使该结构可以工作在一些 频率较低的应用下.

表 3 各锁存器加固能力对比

\begin{tabular}{lcccccccc}
\hline \multicolumn{1}{c}{ 锁存器 } & ST & SR & DT & DR & TT & TR & QT & QR \\
\hline TNURL $^{[9]}$ & $\sqrt{ }$ & $\sqrt{ }$ & $\sqrt{ }$ & $\sqrt{ }$ & $\sqrt{ }$ & $\sqrt{ }$ & $\times$ & $\times$ \\
TNUTL $^{[10]}$ & $\sqrt{ }$ & $\sqrt{ }$ & $\sqrt{ }$ & $\sqrt{ }$ & $\sqrt{ }$ & $\times$ & $\times$ & $\times$ \\
TNU-Latch $^{[6]}$ & $\sqrt{ }$ & $\sqrt{ }$ & $\sqrt{ }$ & $\sqrt{ }$ & $\sqrt{ }$ & $\times$ & $\times$ & $\times$ \\
LCTNUT $^{[11]}$ & $\sqrt{ }$ & $\sqrt{ }$ & $\sqrt{ }$ & $\sqrt{ }$ & $\sqrt{ }$ & $\times$ & $\times$ & $\times$ \\
QNURL & $\sqrt{ }$ & $\sqrt{ }$ & $\sqrt{ }$ & $\sqrt{ }$ & $\sqrt{ }$ & $\sqrt{ }$ & $\sqrt{ }$ & $\sqrt{ }$ \\
\hline
\end{tabular}

综上所述，本文提出的 QNURL 结构虽然在面 积、延迟方面的开销比现存的一些结构大, 但是单 粒子加固能力更强，适用于更小的特征尺寸以及 复杂的环境.

\section{3 衍生结构}

QNURL 结构是基于容忍三点翻转的结构发展 而来的新结构 ${ }^{[14]}$, 还可以按照本文方法继续衍生, 针对任意 $N$ 节点翻转可以按照这样的连接方式衍 生出 $(N+1) \times 2 N$ 的结构框架. 其中, 该框架的单 点结构就是 DICE 结构, 目前这种框架已经通过了 单点、双点、三点、四点翻转的仿真实验. 框架下 结构的具体的性能如表 4 所示. 表中翻转节点数目 表示 $(N+1) \times 2 N$ 中 $N$ 的大小, 在第 1 列列举了在现 行的 CMOS 工艺下常出现的 4 种翻转形式, 并给出 在每种情况下的晶体管数目. 内部节点数目表示 构成锁存器锁存环的节点数, 罙余节点数目表示 在锁存器内部产生节点翻转后, 需要构建多少额 外节点才能将翻转节点的逻辑值自恢复. 穴余节 点比例表示额外节点占节点总量的比例. 从表 4 中 可以看出, 由于该框架采用了双输人反相器使每 种结构的晶体管数目都较少，但同样的原因造成 内部节点数目非常多. 内部节点数目较多会使节 点受到高能粒子的攻击频率上升，但是在另一方 面，圥余的节点会为受到攻击的粒子吸收一部分 的额外电荷, 节点越多越密集, 分担的电荷就越 大，可以使单个节点吸收的电荷量达不到翻转的 阈值, 反而会降低翻转节点的数目. 针对不同的环 境选择框架 $(N+1) \times 2 N$ 中的 $N$ 值, 会使结构的开 销以及加固性能达到最好.
表 4 该框架下的不同结构性能

\begin{tabular}{ccccc}
\hline $\begin{array}{c}\text { 翻转节 } \\
\text { 点数 }\end{array}$ & 晶体管数 & 内部节点数 & $\begin{array}{c}\text { 圥余节 } \\
\text { 点数 }\end{array}$ & $\begin{array}{c}\text { 几余节点 } \\
\text { 比例 }\end{array}$ \\
\hline 单点 & 18 & 4 & 3 & $2 / 3$ \\
双点 & 38 & 12 & 10 & $5 / 6$ \\
三点 & 66 & 24 & 21 & $7 / 8$ \\
四点 & 102 & 40 & 36 & $9 / 10$ \\
\hline
\end{tabular}

双输人反向器是一种面积较小的器件, 但是 它存在的问题是 PMOS/NMOS 管可能同时导通, 虽然可以利用文中的方法得到稳定的输出, 但瞬 时功耗会很大. 为了解决这个问题，可以将提出的 结构中的一部分器件改为堆叠 PMOS 的不对称的 $C$ 单元结构. 其结构如图 9 所示, 由于该结构的真 值表与双输人反相器完全相同, 所以两者工作原 理相同, 两者可以互换. 采用这种方法, 当电路中 发生 SEU 后, 无论逻辑值如何变化, 从电源到地 之间都不会产生贯通电流，也就减少了部分瞬态 功耗, 但是增加了面积, 可以按需进行选择.

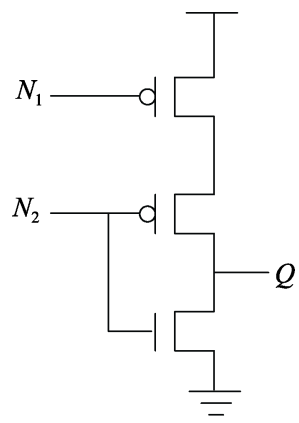

图 9 堆叠 PMOS 的不对称的 $C$ 单元

另外采用图 10 所示堆叠 NMOS 的不对称的 C 单元结构也可以消除部分瞬间功耗. 但是这种 $C$ 单元与双输人反相器的真值表是对称的关系, 即 PMOS 输人是否发生改变才是整个结构输出是否 改变的决定因素. 采用这种方法有 2 种连接方式,

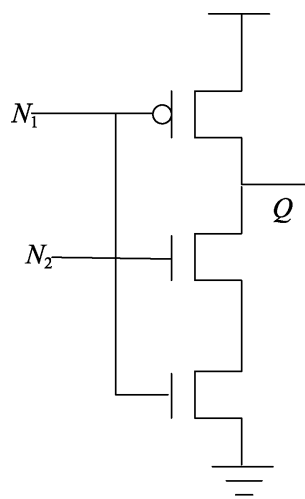

图 10 堆叠 NMOS 的不对称的 $C$ 单元 
第 1 种是将 QNURL 结构中双输人反相器的 NMOS/PMOS 的连接方式交换, 接着按照同样的 连接方式用堆叠 NMOS 的不对称的 $C$ 单元结构替 换电路中所有的双输人反相器. 第 2 种是 QNURL 结构中所有双输人反相器的 PMOS 采用大比例系 数宽长比, NMOS 采用小比例系数宽长比, 使得双 输人反相器的工作原理与堆叠 NMOS 的不对称的 $C$ 单元相同, 这样只需要交换 QNURL 结构中双输 人反相器中 NMOS/PMOS 的连接方式, 按照功能 需求合理选择替换双输人反相器的数目即可.

QNURL 结构在输人端用一个传输门直接将 $D$ 值输出, 这种方法减小了部分延迟, 但是由于 该器件设计在航天领域, 延迟过小可能会使器件 受到 SET 的影响, 为了增大器件容忍 SET 的能力, 可以在 $D$ 与 $Q$ 之间插人一个延迟模块，使该设计 的输人端容忍持续时间更长的扰动脉冲，但是新 加人的延迟结构会增大结构整体的面积、功耗开销, 另外若 SEU 发生在延迟结构处, 也会影响到整个结 构的加固性能, 所以延迟结构的加人要很谨慎.

\section{4 结 语}

本文主要提出了一种可以自恢复 4 个节点同 时翻转的加固锁存器, 并由此衍生了 $(N+1) \times 2 N$ 的结构框架. 这种框架下的结构, 采用双输人反相 器作为基本单元，保证结构在低开销的基础上，可 以自恢复发生翻转的内部节点. 使用 Hspice 进行 仿真分析，证明了该框架的加固能力和低开销性 能. 另外针对该框架下双输人反相器的瞬态功耗 过大的问题, 本文也提出了 2 种牺牲面积指标的替 代方案. 最后指出该框架还可以在输人端加人延 迟逻辑扩大该结构的适用范围，这是下一步研究 的重点.

\section{参考文献(References):}

[1] Ibe E, Taniguchi H, Yahagi Y, et al. Impact of scaling on neutron-induced soft error in SRAMs from a $250 \mathrm{~nm}$ to a $22 \mathrm{~nm}$ design rule[J]. IEEE Transactions on Electron Devices, 2010, 57(7): $1527-1538$
[2] Feng Yanjun, Hua Gengxin, Liu Shufen. Radiation hardness for space electronics[J]. Journal of Astronautics, 2007, 28(5): 1071-1080(in Chinese)

(冯彦君, 华更新, 刘淑芬. 航天电子抗辐射研究综述 $[\mathrm{J}]$. 宇 航学报, 2007, 28(5): 1071-1080)

[3] Jiang J W, Xu Y R, Ren J C, et al. Low-cost single event double-upset tolerant latch design[J]. Electronics Letters, 2018, 54(9): 554-556

[4] Eftaxiopoulos N, Axelos N, Pekmestzi K. DONUT: a double node upset tolerant latch[C] //Proceedings of the IEEE Computer Society Annual Symposium on VLSI. Los Alamitos: IEEE Computer Society Press, 2015: 509-514

[5] Huang Z F, Zhang Y Y, Su Z A, et al. A hybrid DMR latch to tolerate MNU using TDICE and WDICE[C] //Proceedings of the 27th IEEE Asian Test Symposium. Los Alamitos: IEEE Computer Society Press, 2018: 121-126

[6] Watkins A, Tragoudas S. Radiation hardened latch designs for double and triple node upsets[J]. IEEE Transactions on Emerging Topics in Computing, 2020, 8(3): 616-626

[7] Blum D R, Delgado-Frias J G. Schemes for eliminating transient-width clock overhead from SET-tolerant memory-based systems[J]. IEEE Transactions on Nuclear Science, 2006, 53(3): 1564-1573

[8] Calin T, Nicolaidis M, Velazco R. Upset hardened memory design for submicron CMOS technology[J]. IEEE Transactions on Nuclear Science, 1996, 43(6): 2874-2878

[9] Yan A B, Feng X F, Hu Y J, et al. Design of a triple-node-upset self-recoverable latch for aerospace applications in harsh radiation environments[J]. IEEE Transactions on Aerospace and Electronic Systems, 2020, 56(2): 1163-1171

[10] Liu X. Multiple node upset-tolerant latch design[J]. IEEE Transactions on Device and Materials Reliability, 2019, 19(2): 387-392

[11] Yan A B, Lai C P, Zhang Y L, et al. Novel low cost, double-and-triple-node-upset-tolerant latch designs for nano-scale CMOS[J]. IEEE Transactions on Emerging Topics in Computing, 2021, 9(1): 520-533

[12] Eftaxiopoulos N, Axelos N, Pekmestzi K. DIRT latch: a novel low cost double node upset tolerant latch[J]. Microelectronics Reliability, 2017, 68: 57-68

[13] Katsarou K, Tsiatouhas Y. Soft error interception latch: double node charge sharing SEU tolerant design[J]. Electronics Letters, 2015, 51(4): 330-332

[14] Huang Zhengfeng, Guo Yang, Pan Shangjie, et al. Tri-node upset self-recovery latch design in $32 \mathrm{~nm}$ CMOS technology[J]. Journal of Computer-Aided Design \& Computer Graphics, 2020, 32(12): 2013-2020(in Chinese)

(黄正峰, 郭阳, 潘尚杰, 等. $32 \mathrm{~nm}$ CMOS 工艺单粒子三点 翻转自恢复锁存器设计 [J]. 计算机辅助设计与图形学学报, 2020, 32(12): 2013-2020) 